\title{
JÓVENES EN LA CONSTRUCCIÓN DE COMUNIDAD A TRAVÉS DE LA CULTURA LOCAL
}

\author{
Germán Alejandro García Lara' \\ Oscar Cruz Pérez \\ Carlos Alberto López Maza \\ Universidad de Ciencias y Artes de Chiapas, Mexico
}

\begin{abstract}
RESUMEN
El trabajo muestra la participación, organización y experiencia de los jóvenes en el proceso de movilización e involucramiento de los habitantes de los barrios en actividades de promoción del arte y la cultura en un municipio del estado de Chiapas, México. Desde una perspectiva cualitativa se recupera y analiza información surgida en conversaciones informales y entrevistas de siete organizadores y activistas del movimiento Somos Barrio Fest, jóvenes estudiantes y profesionales de entre 16 y 27 años, a quienes les interesa convocar a creadores, artistas y artesanos para integrarse a una cartelera cultural en la vía pública, para promover el arte y la cultura y reconfigurar relaciones comunitarias entre personas del lugar y de fuera de los barrios. Esta experiencia rompe con el estereotipo de que los jóvenes se encuentran inmovilizados ante las necesidades de su entorno y altamente vulnerables a las problemáticas psicosociales.
\end{abstract}

Palabras clave

Psicología comunitaria, jóvenes, cultura popular, cultura local, arte.

\begin{abstract}
The work shows the participation, organization and experience of the young persons in the process of mobilization and involvement of the inhabitants of slum dwellers in activities of promotion of the art and the culture in a municipality from Chiapas. From a qualitative perspective it recovers and analyzes information arisen in informal conversations and interviews of seven organizers and activists of the movement We are Neighborhood Fest, young students and professionals of between 16 and 27 years, to whom they are interested in calling for creators, artists and craftsmen to join to a cultural billboard the public highway, to promote the art and the culture and community relations are re-form between persons and out of the neighborhoods. This experience breaks with the stereotype, that the young persons are immobilized before the needs of his environment and highly vulnerable to the psychosocial problematics.
\end{abstract}

Key words

Community psychology, young people, popular culture, local culture, art.

\footnotetext{
${ }^{1}$ Correspondence about this article should be addressed to German Alejandro Garcia Lara. Email german.garcia@unicach.mx
} 


\section{YOUNG PEOPLE IN THE CONSTRUCTION OF COMMUNITY THROUGH THE LOCAL CULTURE}

Una comunidad puede entenderse como "una unidad geográficamente situada, compacta y homogénea, unida por lazos de cooperación e intereses comunes" (Bang y Stolkiner, 2013, p. 125); no como un lugar definido o limitado, sino como una red de relaciones sociales cuya territorialidad se instituye a partir de la significatividad de dichas relaciones que van más allá de un espacio determinado, que desarrolla y articula ciertas dimensiones económicas, políticas, laborales y culturales que las significan, creando formas específicas de interacción, dinámicas sociales cuyo intercambio de información construye la vida social (Piselli, 2007, Rodríguez y Montenegro, 2016).

No son pocos los actores sociales que con un sentido crítico y propositivo desarrollan trabajos orientados al desarrollo comunitario en los ámbitos educativo y cultural; no obstante, ello resulta fascinante cuando son los jóvenes quienes se organizan para el desarrollo y difusión de su cultura.

Se considera juventud a un periodo de tiempo y condición de los sujetos que están en un proceso de transición de la niñez a la adultez. De acuerdo con Anzaldúa (2006, p. 107), el vocablo juventud es "una categoría histórica, una construcción cultural que alude a la forma en que cada sociedad organiza la transición de los sujetos de la infancia a la edad adulta". Tal concepción va más allá de la referencia a un proceso de instauración identitario ideal como el sugerido en la sociedad occidental. Los jóvenes en la actualidad son un grupo de población que se encuentran escolarizados, empleados o en situación de marginalidad. Estos últimos, padecen la falta de oportunidades escolares y laborales y son colocados en situación de exclusión social, lo que dificulta su desarrollo personal.

En general, muchas de las acciones que describen la participación de los jóvenes se realizan en el marco de las instituciones, no obstante, resulta evidente la emergencia de agrupaciones cuya experiencia comunitaria captura nuevos espacios de acción colectiva.

Font y Blanco (2006) señalan que la participación "es cualquier actividad dirigida a influenciar directa o indirectamente en las políticas" (p. 17), procurando el bienestar de los grupos o sociedades a los que pertenecen; además de esta dimensión politíca, es subversiva y fortalecedora porque "permite a los individuos tener oportunidades para adquirir competencias, desarrollar múltiples funciones en la organización y entrar en contacto con otros miembros de la comunidad" (Ramos y Maya, 2014, p. 171).

De acuerdo con Trilla y Novella (2001), la participación puede tener distintas formas, como la participación simple, en que se está como espectador, la participación consultiva, que supone escuchar la palabra de los otros, la participación proyectiva, en que el sujeto se convierte en agente; y la metaparticipación en que desarrollan "nuevos espacios y mecanismos de participación" (p. 150). Asimismo, establecen ciertos criterios a través de los cuales es posible precisar las formas de participación, estas incluyen la implicación (nivel afectivo), información/conciencia (nivel cognitivo), la toma de decisiones y el compromiso/responsabilidad por el que se asumen las consecuencias de las acciones que realizan.

En la participación comunitaria, “...los individuos se involucran porque de manera colectiva buscan satisfacer las necesidades de sus miembros y colectividad" (Aguirre, 2012, p. 35). Dicha participación puede darse cuando convergen las voluntades y se asumen compromisos y responsabilidades compartidas en torno a un objetivo común, cuyo esfuerzo se manifiesta en las acciones que promueven para el desarrollo de su comunidad. Este proceso, según Font y Blanco (2006), cuenta con cuatro etapas que son la iniciativa, la movilización, la participación y los efectos y resultados.

La participación en la comunidad es mucho más profunda cuando el sujeto forma parte de las decisiones y el curso de las acciones que se realizan, cuando como actor social (Bourdieu, 1993), participa de forma activa en el proceso que se desarrolla en torno al trabajo que encierra dicha participación, incluyendo o haciendo de lado el material o acciones a seguir, interviniendo en las decisiones que se toman en el recorrido del proyecto, forjando relaciones que se establecen entre los colaboradores o significándose el sentido cultural de su praxis.

Como expresa Kloos et al. 2011 (citado por Ramos y Maya, 2014, p. 170): "Al participar en organizaciones locales, los miembros de la comunidad adquieren control sobre su entorno, se identifican con la comunidad y contribuyen a fortalecer la cohesión social", asimismo, es posible conocer del impacto de sus 
acciones en el territorio local y vida cotidiana y con ello, de su comunidad y persona (Agudelo, Murillo, Echeverry y Patiño, 2013), en ámbitos como la formación, el poder o cambio social.

Este proceso posibilita el fortalecimiento de los sujetos, grupos y comunidades, a través de la participación, el apoyo y desarrollo de sus capacidades, teniendo como eje central a la propia comunidad. Bajo esta perspectiva, la denominación de fortalecimiento suple a la de empoderamiento, considerando a este como: "el proceso mediante el cual los miembros de una comunidad (individuos interesados y grupos organizados) desarrollan conjuntamente capacidades y recursos para controlar su situación de vida, actuando de manera comprometida, consciente y crítica, para lograr la transformación de su entorno según sus necesidades y aspiraciones, transformándose al mismo tiempo a sí mismos" (Montero, 2006, p. 72), lo que requiere de la acción conjunta y solidaria de los integrantes de la comunidad.

Las acciones de creación de espacios públicos, de transformación del entorno, constituyen formas de resistencia cultural popular que revitalizan los saberes y tradiciones de la comunidad haciendo confluir diversas manifestaciones y expresiones mediante la socialización de su experiencia. Desde esta óptica los jóvenes estudiantes o artistas que participan en actividades de desarrollo del arte o la cultura, pueden ser considerados como trabajadores culturales o intelectuales públicos (Giroux, 2001), en la medida de que a través de su práctica articulan y redefinen aspectos relacionados con lo educativo y lo político en el espacio de la cultura popular, de la calle, en que recuperan lo propio de la comunidad incorporando formas de mediatización social que dan cuenta de un proceso organizado y colaborativo para el desarrollo activo de las potencialidades de los integrantes de la comunidad y de ellos mismos.

Esta forma parte de la llamada pedagogía cultural, que de acuerdo con R. Williams (citado por Giroux, 2001, p. 133), concibe "la fuerza educativa de toda nuestra experiencia social y cultural... [como un aparato de instituciones y relaciones] que instruye de manera activa y profunda". Tal aseveración coloca a la cultura como una herramienta educativa y política en relación al poder, que da presencia y palabra a las expresiones de grupos sociales marginales.

Tal forma de enseñanza autogestiva, reconoce y afirma el papel político de la educación porque informa e interviene sin olvidar las condiciones socioculturales del entorno en que se realiza. La acción reflexiva que se gesta en este proceso, considera la llamada pedagogía preformativa referida por Grossberg (citado por Giroux, 2001, p. 128) "de entender la teoría como el fundamento para <<intervenir en cada contexto y en el poder... y con ello proporcionar estrategias a las personas para mejorar su contexto $>$ ”. Es por ello, que los espacios en que los líderes y participantes del proyecto discuten, replantean, renegocian o reeelaboran las líneas de acción que se seguirán en el proceso de trabajo comunitario, recupera con pertinencia los conocimientos aprendidos a partir de su experiencia, pero también aquellos desarrollados en su proceso de escolarización.

Con el reconocimiento de las problemáticas comunes, no solo los adultos, sino también los jóvenes se dan cuenta de dichas dificultades y arremeten contra estas a partir de la construcción de un colectivo, cuyo posicionamiento político resiste los embates de una sociedad globalizada, aunado a lo cual, les permite consolidar su estructura psíquica y transitar de la infancia a la vida adulta.

Existen diversos trabajos que dan cuenta del quehacer comunitario por parte de los jóvenes, algunos de estos profundizan en las propuestas comunitarias de tipo pedagógico y de difusión por jóvenes indígenas (Bautista y Juárez, 2016); otros más se realizan en el campo de la salud (Díaz, Loreto y Cumsille, 2003; Bang y Stolkiner, 2013); o de la cultura (Castillo, 2011, Rodríguez y Boada, 2016).

Este tipo de actividades de participación comunitaria, se realizan en México en ciudades como Querétaro con el colectivo Yo Soy 132 como una herramienta de convivencia entre ciudadanos y lo denominan Juanípero Fest; en Monterrey, Tijuana y Ciudad Juárez se desarrolla el proyecto Vivamos la calle a cargo del Programa para la convivencia ciudadana cuyo objetivo es la promoción de comunidades resilientes a los embates del crimen y la violencia, articulando para ello el gobierno, la sociedad civil y las comunidades.

En Chiapas, uno de los proyectos más representativos lo constituye el Somos Barrio Fest, el cual busca generar el involucramiento y participación comunitaria de los jóvenes, fomentar la convivencia entre vecinos del barrio y promover la obra de artistas y artesanos locales; sin embargo, nada se ha escrito sobre este proyecto. En este sentido, el presente trabajo tiene como propósito describir el proceso de participación comunitaria realizado 
por jóvenes en dicho festival, llevado a cabo en un barrio de Ocozocoautla de Espinosa, en el estado de Chiapas, México.

\section{El abordaje metodológico}

La diversidad y pluralidad de la cultura demanda el estudio de la subjetividad, los significados de la experiencia y las prácticas cotidianas (Flick, 2007), situación que en la investigación cualitativa considera el estudio de la oralidad, la particularidad, la localidad y su análisis desde un cierto contexto histórico y social (Toulmin, 2001).

\section{El contexto de estudio y los participantes}

Ocozocuautla de Espinosa, es como cualquier otra pequeña ciudad de Chiapas, la caminata por sus calles, parques y barrios incitan al encuentro con los otros, pero también recuerda las carencias y necesidades que con antelación han expresado miembros de la comunidad. El barrio es una subdivisión de un pueblo o de una ciudad donde sus habitantes comparten aspectos culturales y donde se desarrolla un fuerte sentido de pertenencia. Es un territorio que pertenece a sus habitantes y en el que también los habitantes pertenecen a ese territorio, espacio donde se construyen identidades y se establecen relaciones de convivencia entre ellos.

El municipio del mismo nombre, se localiza en el estado de Chiapas, México. Se constituye por 5 pueblos, 10 colonias, 4 ejidos, 11 rancherías, 4 fraccionamientos y 14 barrios (Vive Mx, 2016). Su población consta de 72,426 personas, de los cuales aproximadamente un $20 \%$ corresponden a una población indígena zoque:; sin embargo, sólo el $2 \%$ de estos hablan una lengua indígena ya que en su mayoría habla el español.

Para el año 2010, cerca del $82 \%$ del total de la población se encontraba en situación de pobreza siendo esta de moderada a extrema. Se le cataloga en condición de rezago educativo y por lo menos el $50 \%$ de la población presenta carencia social tomando en cuenta su precariedad económica, nutricional, de salud y vivienda, además de un elevado índice de marginación (SEDESOL y CONEVAL, 2016).

Los jóvenes ante la escasez de tierras, de trabajo, de oportunidades de estudio y falta de apoyos de proyectos productivos sustentables, emigran hacia el norte del país, Estados Unidos o a Cancún para buscar nuevas oportunidades de vida, sufriendo explotación, discriminación y maltrato.

El Somos Barrio Fest, se ha realizado en el barrio Unión Hidalgo y en el de San Bernabé de la ciudad de Ocozocuautla de Espinosa. Los participantes en la emisión del festival cultural y artístico llevado a cabo en el 2015 en el barrio San Bernabé, son jóvenes entre los 16 y 27 años, estudiantes de preparatoria, universidad y egresados de diferentes carreras profesionales habitantes de la cabecera municipal de Ocozocoautla de Espinosa.

Para la realización del presente trabajo se contactó con los organizadores del festival a quienes se explicó los objetivos, procedimiento y alcance de la presente investigación, solicitando su autorización para la aplicación de entrevistas. Previo consentimiento informado, se llevó a cabo la aplicación de entrevistas y charlas de marzo a octubre de 2015 con siete de los organizadores que tuvieron mayor constancia en las juntas y actividades previas, durante y al final del festival.

\section{Técnicas e instrumento}

La técnica empleada fue el registro de notas a partir de conversaciones informales tenidas con los participantes, además de entrevistas semiestructuradas, las cuales son de diversos tipos, empleándose la de tópico, utilizada para aprender más sobre un evento o tema (Mayan, 2001).

La entrevista semi estructurada se compone de preguntas abiertas preparadas antes de la entrevista siendo una guía para ahondar sobre ciertos temas de interés del investigador, estas preguntas deben ser mínimas para no entorpecer el flujo de la entrevista. La guía de entrevista incluyó los siguientes temas:

- Conocimiento sobre el proyecto.

- Antecedentes de participación en otros proyectos institucionales o comunitarios, motivaciones e intereses para hacerlo.

- Actividades en las que participa.

\footnotetext{
${ }^{2}$ Grupo étnico de la zona norte de Chiapas. A partir de la erupción del volcán Chichonal en 1982, algunos de ellos son reubicados en el municipio de Ocozocoautla de Espinosa. ARTICLES I 48
} 


\section{Análisis de la información}

El análisis de la información, consideró la transcripción a medios digitales de las entrevistas aplicadas, así como de las notas de campo, posteriormente en la fase analítica, se redujeron los datos, codificándose a través de categorías que emergieron del material escritural, los cuales se organizaron en bloques temáticos, que fueron compartidos nuevamente con los entrevistados a fin de retroalimentar su integración, lo que requirió la adecuación menor del análisis realizado, conformándose finalmente el informe de trabajo (Rodríguez, Gil \& García, 1996).

\section{Resultados}

Los bloques temáticos estructurados incluyen la descripción y antecedentes del proyecto y de los agentes participantes, de quienes se delinea su formación académica, profesional y personal, así como su experiencia en proyectos similares; el desarrollo, estrategias y financiamiento con que se realiza el proyecto y la concepción que se tiene de este; las acciones que se realizan para el desarrollo del patrimonio cultural, arte y la convivencia, y la proyección al futuro del Somos Barrio Fest. A continuación se describen cada uno de estos.

\section{Barrio y vía pública: descripción y antecedentes de Somos Barrio Fest}

Somos Barrio Fest es una organización sin ánimo de lucro que genera espacios para artistas y artesanos locales al mismo tiempo que los reconoce mediante un ejercicio de participación comunitaria de los jóvenes, de inclusión y convivencia entre los habitantes del barrio.

Un organizador manifiesta que el Somos Barrio Fest es:

...un festival de barrio que tiene como propósito involucrar y movilizar a la gente del municipio de Ocozocoautla, a creadores, artistas y artesanos para que se integran a una cartelera cultural en la vía pública, para que se de una interacción entre la misma gente del lugar y fuera de los barrios... podemos decir que el Somos Barrio Fest también está promoviendo este turismo entre barrios, este turismo cultural (Alonso).

El proyecto tiene como antecedentes dos proyectos aliados, la Biblioteca callejera que se integra por grupos de jóvenes que fomentan la lectura prestando libros los días domingo en el parque central de Ocozocoautla, y el grupo de teatro Arte y cultura de Ocozocoautla

...el primer encuentro que yo tengo con algunos organizadores fue por un proyecto paralelo que es la Biblioteca callejera, que en cierto modo ha sido una plataforma desde donde se han operado actividades, se han desprendido ideas, ¡fue la semilla! (Alonso).

Los integrantes que precedieron al grupo actual fueron 17 jóvenes, algunos de los cuales participaron en la segunda emisión del mismo.

Las emisiones del festival se desarrollan en los parques de cada barrio ya que:

...es un sitio de referencia para todo el barrio; es decir, cultural y tradicionalmente la plaza sirve como punto de encuentro, como epicentro del barrio (Alonso).

Las actividades que se realizan están basadas en disciplinas artísticas como la pintura, la música y el teatro; incluye el patrimonio cultural intangible del municipio al abordar la historia del barrio, sus tradiciones y el trabajo artesanal del mismo. Además, se implementan actividades para niños como talleres de pintura, teatro y manualidades.

\section{Jóvenes intelectuales públicos}

Los integrantes del Somos Barrio Fest se estructuran en tres tipos de actores: Los organizadores, quienes son jóvenes de 16 a 27 años de edad, estudiantes de bachillerato, universidad y universitarios egresados que se encargan de la planeación, gestión y ejecución del festival. Sin una distinción oficial se dividen por el grado de compromiso y constancia en las actividades, sin embargo, las decisiones se toman en grupo, reconociendo que hay opiniones que tienen más relevancia por ser de organizadores con más experiencia. Este grupo se forma invitando a jóvenes de escuelas preparatorias locales, proyectos de gestión artística o por aquellos que asisten al festival y se interesan en participar. Debido a su actividad escolar y laboral algunos de ellos pasan a ser 
colaboradores, esta dinámica aplica también para los participantes del barrio. Estos últimos constituyen el segundo grupo de actores, quienes son en su mayoría artistas o artesanos que conforman la cartelera cultural y realizan las presentaciones artísticas o la enseñanza en los talleres; a este sector se les contacta de casa en casa con la modalidad de bola de nieve y se limita de seis a diez individuos o grupos. Finalmente, se incluyen a los colaboradores, personas que aportan equipo de sonido, mobiliario o dinero.

Los jóvenes con más experiencia fungen como líderes; sin embargo, buscan involucrar a los demás miembros en las diferentes actividades organizativas, de manera que la toma de decisiones, el curso de las acciones y con ello el poder y la responsabilidad sean compartidos. En este sentido, la participación alcanza un nivel de agencia (Trilla y Novella, 2001).

Los participantes se dividen casi en partes iguales entre los que tienen experiencia en otros proyectos y los que incursionan por primera vez en el mismo, los primeros coinciden en que no habían estado en un proyecto de dimensiones y condiciones similares, sea por el número de participantes, las diversas acciones desarrolladas o porque no habían sido organizadores, aunque algunos refieren cierta experiencia como coordinadores de talleres o integrantes de grupos artísticos en otras organizaciones sociales.

$\mathrm{Su}$ accionar se mueve entre el aprendizaje continuo y el desarrollo de nuevas ideas, a partir de los conocimientos y habilidades con los que cuentan, el asalto de espacios públicos como los parques y el desarrollo de nuevos mecanismos de involucramiento y participación de la población, situación que Trilla y Novella (2001) denominan como metaparticipación. Este aspecto, es expuesto por Montenegro (2004) como una de las características de la participación comunitaria: "Es un proceso educativo no formal que se desarrolla en las relaciones interpersonales establecidas durante la práctica misma de la participación" (p. 86). Al respecto, uno de los entrevistados plantea:

...desde nuestro capital cultural echamos a andar esto como una suerte de aparato teórico, pero también es cierto que no nos dedicamos en realidad como gestores culturales, entonces de manera intuitiva, de manera empírica, lo que hemos hecho hasta ahora ha sido un trabajo de ensayo y error. Desde el inicio en cierto modo teníamos claro que queríamos hacerlo (Alonso).

Sin embargo, algunos voluntarios tienen una formación académica que abona con su conocimiento al proyecto:

...alguno de nosotros tenemos estudios con respecto de las artes, la literatura, la cultura, la psicología, arqueología (Carlos).

Derivado de la experiencia en el proyecto, dos de los participantes han optado por estudiar la licenciatura en gestión y promoción de las artes y la licenciatura en artes visuales. Quienes estudian una disciplina no relacionada con las acciones que se realizan en el proyecto comentan que ello no les limita a participar en las actividades del festival.

Algunos voluntarios manifiestan que entre su formación académica y su labor comunitaria hay bastante discrepancia, aunque consideran que el conocimiento escolar es un punto de apoyo pero no decisivo; para otros, el proyecto les da un horizonte en su aprendizaje académico a partir de una actividad práctica en que se desarrollan conocimientos, habilidades, saberes, que les significa adquirir experiencia útil para su futuro laboral, ver realizada su labor como profesionales o contribuir a su desarrollo personal. Este accionar educativo, forma parte de lo que configura al joven trabajador de la cultura (Giroux, 2001).

\section{El proyecto Somos Barrio Fest, desarrollo y estrategias}

Los organizadores perciben al proyecto como "avanzado, estructurado, original o divertido"; como el inicio de algo más trascendental que puede abonar a un sentido de pertenencia a la comunidad. El proceso de participación se valora como satisfactorio si "la mayor parte de los sectores creen que ha valido la pena, que sirve y que confían en éste más que al principio" (Font y Blanco, 2006, p. 22), además, un aspecto que enfatizan los participantes del Somos Barrio Fest es que el proyecto es novedoso:

...no está bien estéticamente trabajado, pero ¡es algo artístico!, ¡algo nuevo!, ¡algo fluido! (Adin).

El trabajo con niños y adolescentes es una motivación constante, pues se manifiestan responsables de formar una mejor sociedad a través de dichas acciones.

En la realización del proyecto, una fase inicial incluye la negociación, discusión y definición de las líneas de acción, la elección del barrio a intervenir, la valoración de la infraestructura de la plaza y los recursos con los que se cuenta. Este proceso es uno de los momentos más ricos y participativos del proyecto donde se discuten las propuestas y se determina un plan de acción entre todos los integrantes. Al respecto, Maya (2004) escribe: "Las 
personas se sienten más atraídas por los grupos en los que pueden ejercer influencia” (p. 6), por lo que es importante ser tomados en cuenta, que sus ideas cobren peso y puedan cristalizarse en acciones que impacten en otras personas, son esos los alicientes que reciben los organizadores del Somos Barrio Fest:

Sí, lo más importante es que tomen en cuenta mis ideas, mi forma de pensar, que mi opinión y la opinión de mis compañeros influya en otras personas (Mauricio).

Esta parte del proceso se encuentra dentro de la movilización, uno de los cuatro momentos que Font y Blanco (2006) refieren que atraviesa un proceso participativo. Durante la planeación se realiza una esquematización de las tareas más importantes, la fecha para la cual deben estar listas y las personas que las realizarán, lo cual permite "movilizar a los actores políticos y sociales en torno al proceso participativo" (Font y Blanco, 2006, p. 34). Estas tareas se asumen de manera voluntaria, aunque se busca que se cumpla cierto perfil; por ejemplo, los jóvenes más extrovertidos se encargan de difundir el proyecto, los que muestran mayor responsabilidad llevar las finanzas, los que mejor redactan, de elaborar los oficios.

El trabajo de ejecución de la planeación requiere suma coordinación entre los organizadores y los artistas, juntos definen fechas en fines de semana para el día de presentación por ser los días de descanso de escuela y trabajo; para concretar estos primeros encuentros con los artistas se realizan diferentes visitas a sus domicilios. Esta parte del proceso es la que denominan Font y Blanco (2006) como de participación; en esta, la comunicación, el respeto, la disciplina para diferenciar los momentos de trabajo de los de esparcimiento y la amistad son eslabones importantes del proyecto

...es un poco difícil porque también es de escuchar a los demás, qué les parece bien o qué les parece mal, la forma de trabajar las ideas. A veces me parece un poco difícil porque no es tan fácil que las personas tengan una iniciativa, pero luego considero que hay muchas personas que quieren hacer algo o cambiar en algo (Laura).

El festival tiene un tiempo variable de entre dos a tres meses entre los encuentros y reuniones iniciales y su emisión, durante este lapso, hay diversas circunstancias que agilizan o retrasan las actividades; por ejemplo, en algunos barrios se debe solicitar el espacio público a un comité que no es tan conocido y visible para quienes no habitan en ese barrio, en otros la población es más participativa y se agilizan las diligencias.

En dicho proceso, el ejercicio de autocrítica y balance de la actividad es una constante entre los participantes

...en este ejercicio de retrospectiva o de autocrítica o análisis parecía por ejemplo que en realidad no estábamos cubriendo a la totalidad de la población de este barrio, entonces para la segunda emisión que fue en el barrio de San Bernabé, echamos a andar otra estrategia en las reuniones del equipo de logística, dentro del barrio alguien de los habitantes dio prestada la casa para que las reuniones fueran allí, él sirvió entonces como un mediador entre nosotros, como extranjeros del barrio que no vivimos ahí y él que vive en ese lugar. Me parece que eso fue algo novedoso que no se había hecho antes en nuestra organización (Alonso).

Este análisis, se incluye en lo que Font y Blanco (2006) denominan los efectos y resultados de la participación. Dicha innovación es una de las características esperadas en la comunidad después de un ejercicio de participación, fomentando "el surgimiento de nuevas ideas, nuevos modos de hacer y nuevos resultados" (Montero, 2004, p.110).

La estrategia anteriormente citada sirvió para ubicar e invitar con mayor facilidad a los artistas que formarían la cartelera cultural; el siguiente fragmento expone el involucramiento de la población al festival a través de la conexión emocional compartida que se desarrolla entre los participantes:

...escogemos la casa de uno de uno de los integrantes para que nos vaya juntando, para que se integren al proyecto y se desarrolle a nivel más personal una relación de amistad (Adín).

Estos son elementos del fortalecimiento psicológico, la amistad se señala como un pilar importante, en lo que se ha denominado conexión emocional compartida, elemento del sentido psicológico de comunidad, que constituye "el sentimiento de que los miembros importan los unos a los otros y al grupo, una fe compartida de que las necesidades de los miembros serán atendidas mediante su compromiso de estar juntos" (McMillan y Chavis, 1986, p. 9). Tal implicación afectiva, cognitiva y de corresponsabilidad que asumen los participantes por el proyecto (Trilla y Novella, 2001), son el fundamento que posibilita su desarrollo. 
Uno de los retos que enfrenta el proyecto es el de su financiamiento ya que no cuentan con un capital para realizarlo y las actividades no se cobran a los habitantes, siendo esta una de las áreas más difíciles de sortear. Los fondos se consiguen vendiendo postres y dulces antes y durante el festival, mediante patrocinios de empresas locales y gestiones con instituciones estatales. La situación económica y de organización, argumentan los organizadores, dista del conocimiento que adquirieron en la universidad. De la adversidad económica, uno de los entrevistados expresa que anuda el lazo social que existe entre ellos:

...esto hace el lazo más familiar, que se pueda reunir en algún momento (Adín).

La sensación de adversidad y la convivencia refuerzan los lazos emocionales que hay entre los participantes, al respecto Maya (2004) señala que "los miembros reconocen la existencia de un lazo compartido. Este vínculo es el resultado del contacto positivo prolongado y de participar de experiencias y una historia comunes" (p. 6).

\section{Patrimonio cultural, arte y convivencia}

Los ámbitos en los que se intenta influir, se circunscriben a la revaloración de las tradiciones y el patrimonio cultural intangible, el trabajo artístico, la inclusión y convivencia de la población; una manera es invitar a los artistas a ser parte de la cartelera cultural, a los niños de primarias cercanas a que asistan a la plaza del barrio y a los talleres. Con ello, el grupo intenta una recuperación del espacio público y el desarrollo de dinámica social de cooperación e interés común (Bang y Stolkiner, 2013).

...a mi me hablaron que Barrio Fest pretendía amalgamar las partes, digamos las partes perdidas. Hoy en

día, por ejemplo tú vives en tu casa pero no tienes contactos con tus vecinos, antes, prácticamente todo

el mundo conocía a todo el mundo y eso ya no existe (Sara).

La revalorización del trabajo artístico por parte de la población es uno de los propósitos del proyecto:

...lo importante del proyecto es ir metiendo a la gente al ámbito cultural, artístico y que vaya viendo el

valor de cada producto artístico (Laura).

Como se ha señalado, el que las organizaciones culturales o artísticas se involucren en proyectos comunitarios, les hace más propensos a solidarizarse con las comunidades donde laboran y sensibilizarse a las necesidades de estas (Ramos y Maya, 2014; Lechón, 2015); las acciones de difusión de la cultura y el fortalecimiento de la comunidad son consecuencias de dicha acción educativa (Giroux, 2001).

\section{Proyección a futuro del proyecto}

La reflexión en los participantes escudriña sobre su lugar en el mundo, de su corresponsabilidad social, de ser solamente a través del otro, de la utopía que posibilita el cuestionamiento:

¿El Somos Barrio Fest habrá sido ese programa lleno de paja que se ofrecía cada fin de semana en el pueblo, un formato más o hubo algo ahí? Ese destello que cambió el rumbo de la gota de agua generó otra cosa. Quiero decir, ¿qué donación... a la vida podemos hacer desde el Somos Barrio Fest? Y eso transciende ipor mucho!, ipor mucho!, ¡por mucho!, los intereses personales de cada uno de nosotros (Alonso).

Este es un elemento crucial de la participación que se concibe como un espacio dinámico "en el que se reflexiona y actúa colectivamente para la mejora de las condiciones de vida de los miembros de una comunidad" (Montenegro, 2004, p. 85) trascendiendo los intereses individuales.

En esta proyección a fututro se piensa al Somos Barrio Fest como un proyecto con fronteras que se agotarán un día y habrá que moverlas o desplazarlas a otros sitios, así como revalorar sus objetivos y ampliar la apropiación de espacios públicos. Para ello, han considerado realizarlo en colonias o en las zonas periféricas de la cabecera municipal, de innovación para que la población sea cautivada. El proyecto es una apuesta que hace la juventud por sí misma a futuro.

En un promedio de dos a tres años se espera que el Somos Barrio Fest:

...ya se conozca por lo menos en todo Ocozocoautla y tan siquiera una o dos veces ir a a presentarlo a la capital de Chiapas que es Tuxtla Gutiérrez (Mauricio).

Parte de las ambiciones a futuro es tener el poder de convocatoria y la audiencia de un festival realizado por una institución gubernamental llamado Festival de las Artes en Ocozocoautla de Espinosa e inclusive realizar festivales simultáneos en diferentes barrios del municipio. Se espera que el proyecto se torne más complejo y pueda influir en otras áreas como el deporte, la salud, la nutrición, la economía, la convivencia familiar, entre otros temas.

ARTICLES I 52 
Cuando en los jóvenes confluyen acciones de carácter educativo, amalgamadas en torno de problemáticas sociales que se aúnan a otros proyectos relacionados con la preservación o difusión de la cultura, la figura del joven intelectual emerge como vía de mediatización social.

Cada integrante tiene ideas, intereses y proyectos afines y diversos, por lo que ven a Somos Barrio Fest como un punto de articulación, una plataforma para futuros proyectos, un punto de encuentro, de cooperación, de enriquecimiento del trabajo comunitario e ideas, incluso los voluntarios se perciben como formadores a futuro y posibles colaboradores de otros colectivos. Sobre ello, Montenegro plantea que la participación no es un estado estable si no dinámico, así "los sujetos involucrados se forman y forman a otras personas en el manejo de conocimientos y destrezas que dependen de la naturaleza de la experiencia participativa" (Montenegro, 2004, p. 86); este proceso de formación se aprecia al ser un grupo inicial el que forma a otros voluntarios que ahora se muestran interesados en desarrollar sus propias iniciativas y formar a otros.

\section{Discusión}

La comunidad como territorio emblemático del Somos Barrio Fest, ha establecido entre sus actores una red de relaciones en que la cultura constituye el amasijo por el que se teje una dinámica de cooperación, de lucha y significación de la colectividad. De particular relevancia en este proceso, es que sean los jóvenes quienes asuman un protagonismo poco usual y establezcan rupturas con la vivencia infantil a partir de una experiencia en que se hacen cargo de sí, para coincidir con los otros y colaborar en un proyecto común, puerta abierta a la vida adulta que se impregna por una acción participativa en que forjan la transformación de su entorno, una práctica política y educativa en que como intelectuales públicos potencian los saberes y tradiciones de la localidad.

El reconocimiento de los saberes y tradiciones comunitarias, es el principio por el cual se revitaliza y se coloca en un lugar preeminente a la cultura y sus artistas, tal proceso, imbuido de una apasionada actividad nutrida por lazos de amistad, sensibiliza de las problemáticas de la comunidad y genera una respuesta política que irrumpe el barrio que resiste a la globalización de la cultura, su comercialización y consumo sin identidad ni pertenencia.

Las producciones artísticas no tienen como objetivo la venta o comercio de los productos, sino mostrarse, dar cuenta de contenidos simbólicos propios de la cultura local. Esta actividad se opone a una política pública del Estado de la que no son protagonistas o beneficiarios, de una visión globalizada de la vida social y cultural que configura una industria atravesada por la mercantilización, consumo y plusvalía obtenida de su comercialización, que deja de lado el sentido de lo humano, de su historia y territorio emergentes en la participación comunitaria. En este proceso, un primer punto de tensión es justamente el otorgar un sentido político y de acción social transformadora a la actividad cultural y tradiciones de la comunidad local, que contrasta con la visión homogeneizante, universal y superflua con que el Estado asume su cobijo y regulación, con el rótulo de cultura primitiva o expresiones del folclore; por otra parte, es de hacer notar que si bien los trabajos no se alojan en dichas políticas o programas gubernamentales, hacen uso marginal de estos para obtener apoyos materiales, así como de empresas locales que patrocinan ciertas acciones, tal proceder advierte la contradicción entre las formas de autogestión y la reproducción del orden dominante a través de la difusión de los productos de dichas empresas.

Los extremos de un quehacer político de autoorganización comunitaria se empalman en los ciernes de autoestructuración psíquica que redefine su ser como jóvenes. La autogestión inicia con el proceso de incorporación voluntaria al grupo de trabajo, en cuyo seno se planifica y discute la organización del festival, posteriormente, uno de los momentos culminantes es la elección de los espacios de presentación, los parques, las plazas, esto es, el espacio público que se contrapone a los espacios cerrados de la sala municipal o de la casa de la cultura local. Esta visibilización de la cultura en un espacio común es favorablemente acogida por los propios habitantes de la comunidad, en que al menos uno de cada cinco proviene de la etnia zoque, o bien han emigrado de poblaciones cercanas, situación que conlleva a la construcción de un sentido identitario, de pertenencia a la comunidad, que les lleva a acometer la recuperación material y simbólica de aquello que han sido despojados.

En la pedagogía cultural, la presencia y voz de grupos marginales se constituye a través de la animación socio cultural, proyecto humanizador del barrio que tiende a la comunicación intersubjetiva, el aprender a aprender y la potenciación de la experiencia convivencial a partir de una presencia estética y ética para 
comprender y transformar las realidades culturales. Como acción pedagógica da cuenta de aprendizajes humanos, de un saber hacer respecto de lo educativo que transita en el andamiaje de la cultura del barrio.

Lo que se aprende para ser más humano, forma parte de una práctica que pervive por ensayo y error, no son los saberes académicos que irradian las formas de organización, sino la voluntad por participar; y es la experiencia en la interrelación compartida en que emerge la necesidad de un cierto aprendizaje. Los saberes comunitarios y los académicos conformados a partir del proceso de escolarización en que algunos de los jóvenes se encuentran se colocan en común; así, se produce una cierta teorización de la experiencia, que no de la teoría hecha práctica.

Pero hacer experiencia del conocimiento de la comunidad, no es posible sin colocar en tregua lo conocido, los aprendizajes académicos, aquellos que señalan un proceder a priori sin considerar la realidad, ante ello, la experiencia se diluye, pero emergen nuevos conocimientos (Benito, 2017), la posibilidad de experiencias bajo la tutela de lo que el grupo o comunidad consideran como cierto. Este proceso indeterminado, forma intelectuales públicos cuya práctica define lo educativo y político en el escenario de la cultura.

El proceso de negociación, discusión y definición de las líneas de acción, son mecanismos para la toma de decisiones que conlleva al ejercicio del poder, que alcanza una forma de metaparticipación (Trilla y Novella, 2001), en los jóvenes, tal proceso es mediado por su participación como organizadores o colaboradores, función supeditada a la experiencia previa y compromiso en estos eventos. En el caso de los organizadores, la planeación del trabajo supone un proceso de diálogo y consenso con artistas y colaboradores, no exento de conflicto, cuyo desafío es el parteaguas en la conformación de micropolíticas organizativas, aprendizaje que alienta procesos formativos de más largo alcance. Este proceso no es homogéneo ni equilibrado, por el contrario, es conflictivo, diverso, supone un encuentro con los otros, pero también un reconocimiento de las diferencias (Rodríguez y Montenegro, 2016), lo que construye una identidad no clausurada en sí misma, sino pujante, en que se establecen rupturas que dan cuenta de cambios en los sujetos, los grupos y la propia comunidad.

La trascendencia de recuperar el patrimonio cultural intangible, como las tradiciones de la comunidad, la historia del barrio y el trabajo artesanal otorga un lugar primodial a la expresión creadora de grupos marginales, lo que coloca al centro de la acción política a la cultura y a sus agentes.

\section{Conclusiones}

El proceso de participación de Somos Barrio Fest, aprecia un sentido de participación responsable, en que se involucran distintos agentes, los propios participantes, los artistas, artesanos y la población en general.

La labor de participación comunitaria desarrollada es un proceso educativo no formal, incluyente, dinámico en que se busca el logro de metas compartidas, vinculada a un clima de democracia participativa, que se identifica con lo escrito por Montero (2004): "Un proceso organizado, colectivo, libre, incluyente, en el cual hay una variedad de actores, de actividades y de grados de compromiso, que está orientado por valores y objetivos compartidos, en cuya consecución se producen transformaciones comunitarias e individuales" (p. 109).

El proceso de participación de Somos Barrio Fest se impulsa a través de unos jóvenes a otros, de la invitación a los artistas para que integren la cartelera cultural y con los pobladores al invitarlos a asistir; se visibiliza y difunde entre los pobladores, incentivándoles a participar y convivir con otros habitantes del barrio; así, durante su emisión, con la actuación de los artistas y artesanos y la asistencia de los pobladores, es posible el fortalecimiento de los organizadores, la preservación y difusión de la cultura, la participación de la comunidad en su conjunto y la convivencia entre ellos.

La influencia es también un tema medular dentro del grupo ya que relatan que sus opiniones son tomadas en cuenta por los demás, como expresión política y manifestación del poder que emerge de su interacción y trabajo comunitario (Maya, 2004; Meza, 2009).

Montero (2006) plantea que el fortalecimiento “...es un logro de la reflexión, conciencia y acción de las personas interesadas" (p. 62), aspecto ampliamente apreciado en la participación de los jóvenes, lo que les permite definir y realizar con mayor responsabilidad sus actividades escolares o laborales, el superar una ruptura amorosa, ser más sociables, decidir sobre su futuro académico y profesional así como decidir iniciar proyectos por su propia cuenta para atender distintas demandas socioculturales de su comunidad.

En México falta aún camino por recorrer para ampliar la participación de los jóvenes, no obstante, las experiencias que se desarrollan, muestran fehacientemente el sentido de comunidad y el fortalecimiento de los individuos y grupos. 


\section{Referencias}

Agudelo-Ramírez, A., Murillo-Saá, L., Echeverry-Restrepo, L. y Patiño-López, J. A. (2013). Participación ciudadana y prácticas políticas de jóvenes en la cotidianidad. Revista Latinoamericana de Ciencias Sociales, Niñez y Juventud, 11(2), 587-602.

Aguirre Sala, J. F. (2012). La participación ciudadana mediática para descentralizar al Estado (Citizenship.com.2.0). Araucaria. Revista Iberoamericana de Filosofía, Política y Humanidades, 15(29), 33-53.

Anzaldúa Arce, R. E. (2006). Jóvenes frente al abismo. Tramas 24, pp. 105-134. UAM-X, México. Recuperado de: http://www.peu.buap.mx/web/seminario_cultura/Jovenes_frente_abismo.pdf

Bang, C. y Stolkiner, A. (2013). Aportes para pensar la participación comunitaria en salud/salud mental desde la perspectiva de redes. Ciencia, Docencia y Tecnología, XXIV, 46, 123-143.

Bautista Martínez, E. y Juárez López, I. I. (2016). Formas emergentes de participación comunitaria. Los jóvenes indígenas en dos municipios de Oaxaca. El Cotidiano, 197, 102-112.

Benito, K. (2017). Autogestión cultural en la ciudad de Buenos Aires. European Review of Artistic Studies, 8(1), 74-91.

Bourdieu, P. (1993). Cosas dichas. Barcelona: Gedisa.

Castillo, S. (2011). Espacios de arte y cultura en los Centros de Desarrollo Comunitario (CDC). Alteridad, Revista de Educación, 6(2), 127-134.

Díaz, D., Loreto Martínez, M. y Cumsille, P. (2003). Participación comunitaria en adolescentes: Desafíos para la promoción de la salud. Revista de Psicología, XII, 2, 53-70.

Font, J. y Blanco, I. (2006). Polis, la ciudad participativa. Participar en los municipios: ¿quién, cómo y por qué? Papers de Participació Ciutadana, Barcelona: Centre per a la Participació Ciutadana, Organisme Autònom Flor de Maig, Diputació de Barcelona. Recuperado de: https://www1.diba.cat/uliep/pdf/36525.pdf

Flick, U. (2007). Introducción a la investigación cualitativa (2a edición). España: Ediciones Morata.

Giroux, H A. (2001). Cultura, política y práctica educativa (1 ${ }^{\mathrm{a}}$ edición). España: Graó.

Lechón, D. (2015). Sujetos políticos emergentes en espacios urbanos. El caso de El Paliacate en San Cristóbal de Las Casas, Chiapas. Tesis de maestría. San Cristóbal de Las Casas, Chiapas, México: UNICACH.

Maya-Jariego, I. (2004). Sentido de comunidad y potenciación comunitaria. Apuntes de Psicología, 22 (2), 187 211.

Mayan, M. (2001). Una introducción a los métodos cualitativos: Módulo de entrenamiento para estudiantes y profesionales (Trad. César A. Cisneros Puebla). Canadá: Qual Institute Press. International Institute for Qualitative Methodology. Recuperado de: http://www.ualberta.ca/ iiqm//pdfs/introduccion.pdf

McMillan, D. W. y Chavis, D. (1986). Sense of community: a definition and theory. Journal of Community Psychology, 14, 6-23.

Meza Rivera, G. (2009). Comunidad y sentido de comunidad. La intervención del Programa Puente en seis familias en situación de extrema pobreza de la Comuna de La Florida. Santiago. Memoria para optar al título de Psicólogo. Universidad de Chile, Facultad de Ciencias Sociales, Escuela de Psicología. Recuperado de: http://repositorio.uchile.cl/tesis/uchile/2009/cs-meza_g/pdfAmont/cs-meza_g.pdf

Montero, M. (2004). Introducción a la psicología comunitaria. Desarrollo, conceptos y procesos. Buenos Aires, Argentina: Paidós.

Montero, M. (2006). Teoría y práctica de la psicología comunitaria: la tensión entre comunidad y sociedad $\left(1^{\mathrm{a}}\right.$ edición, $3^{\mathrm{a}}$ reimp.). Buenos Aires: Paidós.

Montenegro Martínez, M. (2004). La investigación acción participativa. En G. Musitu Ochoa, J. Herrero Olaizola, L. Cantera Espinosa y M. Montenegro Martínez, Introducción a la Psicología Comunitaria (pp. 78-97). Barcelona, España: Ed. UCO.

Piselli, F. (2007). Communities, places and social networks. American Behavioral Scientist, 50(7), 867-878. 
Ramos-Vidal, I. y Maya-Jariego, I. (2014). Sentido de comunidad, empoderamiento psicológico y participación ciudadana en trabajadores de organizaciones culturales. Psychosocial Intervention, 23, 169-176. Recuperado de: http://dx.doi.org/10.1016/j.psi.2014.04.001

Rodríguez Gómez, G., Gil Flores, J. y García Jiménez, E. (1996). Metodología de la investigacion cualitativa . Granada, España: Ediciones Aljibe.

Rodríguez, A. R. y Montenegro, M. (2016). Retos contemporáneos para la psicología comunitaria: Reflexiones sobre la noción de comunidad. Interamerican Journal of Psychology, 50(1), 14-22.

Rodríguez-Mancilla, M. y Boada, M. J. (2016). Investigación-acción-participativa y renovación urbana en el barrio patrimonial La Ronda de Quito. Interamerican Journal of Psychology, 50(1), 86-95.

SEDESOL / CONEVAL (2016). Informe anual sobre la situación de pobreza y rezago social, Ocozocoautla de Espinosa. Recuperado de: http://www.monitor-odm.chiapas.gob.mx/odm2/wpcontent/downloads/Indicadores/2014_Informe_Sobre_Situacion_Pobreza/Chiapas_Ocozocoautla_de_Espi nosa.pdf [Fecha de consulta: 11 de diciembre de 2016].

Trilla, J. y Novella, A. (2001). Educación y participación social de la infancia. Revista Iberoamericana de Educación, 26, 137-164.

Toulmin, S. (2001). Cosmópolis: el trasfondo de la modernidad. Barcelona: Península.

Vive Mx (2016). Listado de colonias en Ocozocoautla de Espinosa, Chiapas. Recuperado de: https://www.vivemx.com/cols/ocozocoautla-de-espinosa.htm [Fecha de consulta: 11 de diciembre de 2016]. 